\title{
The cerebellum is involved in predicting the sensory consequences of action
}

\author{
Sarah-J. Blakemore, ${ }^{\text {CA,2 }}$ Chris D. Frith and Daniel M. Wolpert' \\ Wellcome Department of Cognitive Neurology and 'Sobell Department of Neurophysiology, Institute of Neurology, University \\ College London, Queen Square, London WCIN 3BG, UK \\ ${ }^{2}$ Present Address: INSERM Unit 280, Mental Processes and Brain Activation, I5I Cours Albert-Thomas, 69424 Lyon Cedex 3 , \\ France \\ ${ }^{\mathrm{CA}}$ Corresponding Author
}

Received 2I March 200I; accepted II April 200 I

\begin{abstract}
We used $\mathrm{H}_{2}{ }^{15} \mathrm{O}$ PET to examine neural responses to parametrically varied degrees of discrepancy between the predicted and actual sensory consequences of movement. Subjects used their right hand to move a robotic arm. The motion of this robotic arm determined the position of a second foam-tipped robotic arm, which made contact with the subject's left palm. Using this robotic interface, computer
\end{abstract}

controlled delays were introduced between the movement of the right hand and the tactile stimulation on the left. Activity in the right lateral cerebellar cortex showed a positive correlation with delay. These results suggest the cerebellum is involved in signalling the sensory discrepancy between the predicted and actual sensory consequences of movements. NeuroReport 12:1879-1884 (C) 2001 Lippincott Williams \& Wilkins.

Key words: Cerebellum; Forward model; Internal model; PET; Prediction; Sensorimotor control

\section{INTRODUCTION}

It has been proposed that information about our motor commands is used to distinguish the sensory consequences of our own actions from externally produced sensory stimuli [1-3]. In order to achieve this, some kind of central monitor [1] or internal forward model (Fig. 1) has been postulated [2,4]. Forward models capture the forward or causal relationship between actions and their sensory consequences by using an efference copy of the motor command to make a prediction of the sensory consequences of the movement. This sensory prediction is compared with the actual consequences of the movement and, depending on its accuracy, can be used to filter sensory information. Self-produced sensations can be accurately predicted on the basis of motor commands and as a result there will be a low level of sensory discrepancy resulting from the comparison between the predicted and actual sensory feedback. This accurate prediction can be used to cancel the ensuing sensation. In contrast, externally generated sensations cannot be predicted on the basis of efference copy and will therefore be associated with a higher level of sensory discrepancy. Such a system can be used to cancel out or attenuate sensations induced by self-generated movement, thereby accentuating sensations originating externally, such as contact with objects.

We have previously demonstrated that the perception of a self-produced sensation is related to the accuracy of the sensory prediction made by the forward model. Using a robotic interface we firstly demonstrated that self-produced and externally produced tactile sensations are perceived differently [5]. Subjects consistently rated a self-produced tactile sensation on their right palm as being significantly less 'tickly', intense and pleasant than an identical stimulus produced by a robot, supporting the demonstration that people cannot tickle themselves [6]. Second, we examined the perceptual effects of altering the correspondence between self-generated movement and its sensory consequences. This was achieved by introducing delays of 100, 200 and $300 \mathrm{~ms}$ between the movement of the left hand and the tactile stimulus on the right palm. In a further condition, trajectory rotations of $30^{\circ}, 60^{\circ}$ and $90^{\circ}$ were introduced between the direction of the left hand movement and the direction of the tactile stimulus on the right palm. The result of increasing the delay or trajectory rotation was that the sensory stimulus no longer corresponded to that which would be normally expected based on the efference copy. Therefore as the delay or trajectory rotation is increased, the sensory prediction becomes less accurate. The results showed that subjects reported a progressive increase in the 'tickly' rating as the delay was increased between $0 \mathrm{~ms}$ and $200 \mathrm{~ms}(p<0.0005)$ and as the trajectory rotation was increased between 0 and $90^{\circ}$ $(p<0.01)$. These results suggest that the perceptual attenuation of self-produced tactile stimulation is due to a precise attenuation of the sensory stimulation, based on specific sensory predictions, rather than a non-specific attenuation of all sensory signals.

The perceptual attenuation of self-produced tactile sensations could be due to gating of activity in somatosensory cortex. Neurophysiological data demonstrate that neuronal 


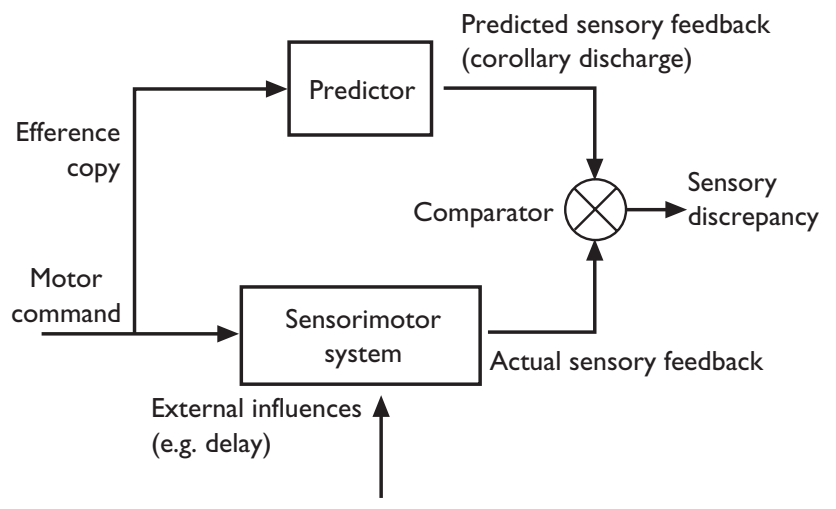

Fig. I. A model for determining the sensory consequences of a movement. An internal forward model makes predictions of the sensory feedback based on the motor command. These predictions are then compared to the actual sensory feedback to produce the sensory discrepancy signal. Self-produced sensations can be correctly predicted on the basis of the motor command, and there will therefore be little or no sensory discrepancy between predicted and actual sensory feedback. As the sensory discrepancy increases (for example by introducing a delay between movement and its sensory consequences) so does the likelihood that the sensation is externally produced. By using such a system it is possible to cancel out the effects on sensation induced by self-motion and thereby distinguish sensory events due to self-produced motion from the sensory feedback caused by the environment, such as contact with objects.

responses in somatosensory cortex are attenuated by selfgenerated movement [7]. Using fMRI we examined the neural basis of the differential perception of self- and externally produced tactile stimuli in humans [8]. We found an increase in activity of the secondary somatosensory cortex and the anterior cingulate gyrus when subjects experienced an externally produced tactile stimulus on their left palm relative to a self-produced tactile stimulus. In order for somatosensory cortex activity to be attenuated to self-produced sensory stimuli, these stimuli need to be predicted accurately. The cerebellum is a likely site for a forward model that provides predictions of the sensory consequences of movements, which are compared with the actual sensory feedback from movements. This hypothesis has been supported by neurophysiological $[9,10]$, functional imaging [11] and computational [12,13] data. Functional imaging studies have shown that distributed regions in the cerebellar cortex are activated during motor learning $[11,14,15]$. One of the main proposed uses of a forward model is for motor learning, which can be achieved by comparing the predicted consequences of an action to the actual result and updating the prediction accordingly [16].

In our fMRI study, there was less activity in the right cerebellar cortex associated with a movement that generated a tactile stimulus than with a movement that did not [8]. This pattern suggests that activity in the cerebellum depends on the specific sensory consequences of a movement. We therefore proposed that the cerebellar cortex might be involved in signalling the sensory discrepancy between the predicted and actual sensory feedback. The aim of the present study was to test this hypothesis directly. To this end, PET was used to examine the brain responses to parametric increases in the level of sensory discrepancy resulting from the comparison between the predicted and actual sensory feedback of movements. To achieve this, a robotic interface was used to vary the correspondence between self-produced movements and their sensory consequences. Subjects were scanned while producing a tactile sensation on the palm of their left hand, by moving a robotic arm with their right hand. By using two robots so that the tactile stimulus could be delivered under remote control by the subject, delays of approximately 0, 100, 200 and $300 \mathrm{~ms}$ were introduced between the movement of the right hand and the tactile stimulus on the left palm. In all conditions the motion of the right hand determined the tactile sensation on the left palm. Only the temporal correspondence between the action of the right hand and the sensory effect on the left palm was altered. The $0 \mathrm{~ms}$ condition corresponds to the normal situation in which subjects use their right hand to move a physical rod across the palm of their left hand. As the delay is increased the resemblance to the normal situation decreases, and hence the forward model's sensory prediction becomes increasingly inaccurate. Thus brain activity that correlates with the delay must be associated with the sensory discrepancy between the predicted and actual sensory feedback from the movement. Based on the proposal that the cerebellar cortex signals the sensory discrepancy between the predicted and actual sensory feedback, it was predicted that blood flow in the cerebellar cortex would increase as the delay increases.

\section{MATERIALS AND METHODS}

Subjects: Six healthy right-handed male volunteers (mean age 24 years; age range 8 years) gave informed consent for the study, which was approved by the National Hospital for Neurology and Neurosurgery Ethics Committee. Consent was obtained from all subjects prior to participation according to the declaration of Helsinki. Permission to administer radioactive substances was obtained from the Administration of Radioactive Substances Advisory Committee (ARSAC) UK.

Procedure: Subjects lay supine in the scanner with their left forearm laid on a flat surface with the palm vertical. Subjects gripped a lightweight rod (radius $0.5 \mathrm{~cm}$ and length $4 \mathrm{~cm}$ ) with the thumb and index finger of their right hand. This rod was attached to an optical encoder (Phantom Haptic Interface, Sensable Devices, Cambridge, MA, USA), and subjects were required to move it sinusoidally (frequency $2 \mathrm{~Hz}$ and amplitude $1.5 \mathrm{~cm}$ ) in the vertical direction. The motion of this rod determined the position of a torque motor, on which was mounted a tactile stimulus constituting a piece of soft foam, which made contact with the subject's stationary left palm (Fig. 2). The motion of the rod held in the right hand was transmitted to the foam-tipped motor thereby ensuring that the motion of the right hand determined the tactile stimulation on the left palm. The robot was controlled by a PC and its position was updated at $1 \mathrm{kHz}$. By delaying the transmission between the encoder and torque motor, computer controlled delays of $0,100,200$ and $300 \mathrm{~ms}$ were introduced between the movement of the right hand and the movement of the tactile stimulus on the left, constituting the four experimental conditions. Subjects practiced the sinu- 


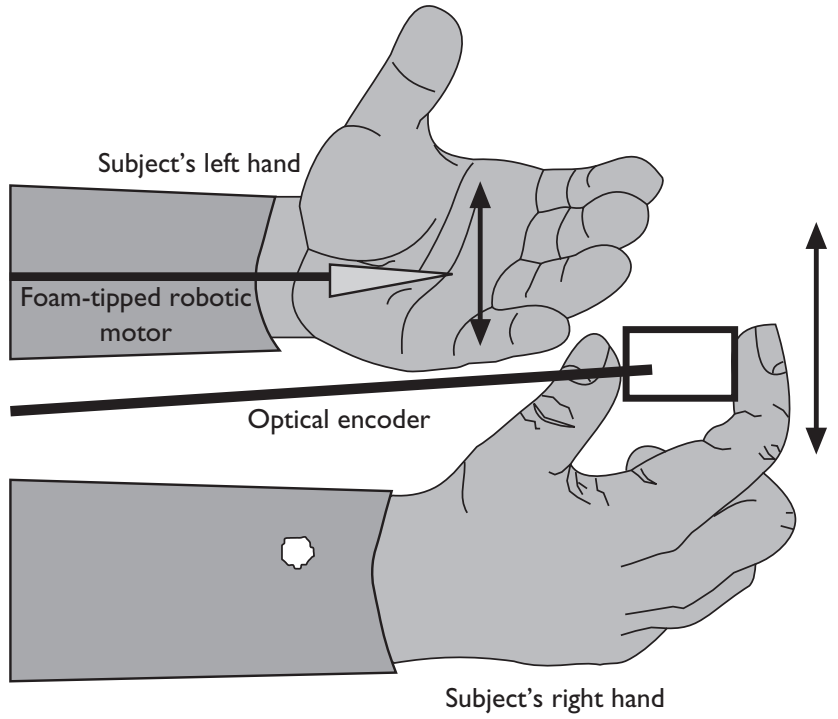

Fig. 2. Diagram of experimental set-up. The subject gripped a lightweight rod with the thumb and index finger of their right hand. This was attached to an optical encoder and subjects were required to move it sinusoidally (frequency $2 \mathrm{~Hz}$ and amplitude $1.5 \mathrm{~cm}$ ) in the vertical direction. The motion of this robot determined the position of a foam-tipped robotic motor touching the subjects' left palm. Thus, the motion of the right hand determined the tactile stimulation on the left palm. In all conditions, subjects were required to move the rod held in their right hand sinusoidally, which, via the two robots, produced the same movement of the tactile stimulus on their left palm. By delaying the transmission between the encoder and robotic motor, computer controlled delays of $\sim 0,100,200$ and 300 ms were introduced between the movement of the right hand and the movement of the tactile stimulus on the left, constituting the four experimental conditions.

soidal movement until they were proficient at producing it. Subjects were informed that the movement of their right hand would produce the movements of the tactile stimulus on their left palm in each condition. However, subjects were not informed of the presence of delays between the movement and the tactile stimulation. Each subject underwent 12 PET scans in a single session, comprising three replications of each of the four conditions. The order of conditions was randomised and counterbalanced within and between subjects. Scanning took place in a darkened room and subjects were asked to keep their eyes closed throughout.

Data acquisition: All subjects underwent both PET and MRI scanning. A Siemens VISION (Siemens, Erlangen) operating at $2.0 \mathrm{~T}$ was used to acquire axial $\mathrm{T} 1$ weighted structural images for anatomical coregistration. PET scans were performed with an ECAT EXACT HR+ scanning system [CTI Siemens, Knoxville, TN] in 3D mode with septa retracted. The axial field of view was $155 \mathrm{~mm}$, providing whole brain coverage including cerebellum. A venous cannula to administer the tracer was inserted in an antecubital fossa vein. Approximately $350 \mathrm{MBq}$ of $\mathrm{H}_{2}{ }^{15} \mathrm{O}$ in $3 \mathrm{ml}$ normal saline were loaded into i.v. tubing and flushed into subjects over $20 \mathrm{~s}$ at a rate of $10 \mathrm{ml} / \mathrm{min}$ by an automatic pump. After a delay of $\sim 35 \mathrm{~s}$, a rise in counts could be detected in the head that peaked $30-40$ s later (depending on individual circulation time). The interval between successive administrations was $8 \mathrm{~min}$. The data were acquired in one $90 \mathrm{~s}$ frame, beginning $5 \mathrm{~s}$ before the rising phase of the head curve. Correction for tissue and helmet attenuation was made using a transmission scan from ${ }^{68} \mathrm{Ga} /{ }^{68} \mathrm{Ge}$ sources at the start of the scanning session. Images were reconstructed by filtered back projection (Hanning filter, cut off frequency 0.5 cycles/pixel) into 63 transverse image planes (separation $2.4 \mathrm{~mm}$ ) and into a $128 \times 128$ pixel image matrix, with a resulting pixel size of $2.4 \times 2.1 \times 2.1 \mathrm{~mm}$, and a resolution of $6 \mathrm{~mm}$ at full width half maximum.

Statistical analysis: Functional imaging analysis used the technique of statistical parametric mapping, implemented in SPM99 (http://www.fil.ion.ucl.ac.uk/spm). For each subject, a set of 12 PET scans was realigned and then stereotactically normalised [17] into the space of Talairach and Tournoux [18]. The scans (72 in total) were then smoothed with a Gaussian kernel of $12 \mathrm{~mm}$ full-width half maximum.

The analysis of functional imaging data entails the creation of statistical parametric maps that represent a statistical assessment of condition-specific effects hypothesised by the experimenter [19]. The effects of global changes in blood flow between conditions were modelled as a confound using a subject-specific ANCOVA. SPM99 was used to identify brain areas where activity was predicted by delay between the movement and the tactile stimulation. To this end, delay, as recorded by a computer, was used as a covariate and regression with this covariate was calculated for every voxel in the whole brain. The amplitude and frequency of the movements in all conditions, which were recorded by the computer, were modelled as confounds in the analysis. Therefore rCBF that was related to movement frequency or movement amplitude was removed. The significance of the regression was displayed in a SPM[t] map, which was then transformed into an $\operatorname{SPM}\{Z\}$ and thresholded at a Z-score of 3.09 $(p<0.05$ uncorrected). We carried out a small volume correction (SVC) [20] on the $p$ values of the ensuing maxima on all predicted brain regions. We report only those that survive this SVC (radius $7 \mathrm{~mm}$, corresponding to the spherical region of the right cerebellum predicted from our previous fMRI results [8]) at $p<0.05$.

\section{RESULTS}

Regions that showed a positive covariance between rCBF and delay: The results of the covariate analysis demonstrate brain regions whose rCBF shows a positive regression on delay between the movement of the right hand and the tactile stimulation on the left palm, after rCBF that relates to movement frequency and movement amplitude was removed. The regression analysis from all six subjects combined demonstrated a significant $(Z=2.82 ; p<0.05$ SVC) positive regression on delay in the middle right cerebellar cortex (coordinates $38,-56,-34$ in Talairach and Tournoux [18]; margin of VI and Crus I in Schmahmann et al. [21]). In single subject analyses all six subjects showed this positive relationship between delay and activity in the right cerebellum. However in one subject this right cerebel- 
lar activation was only present at a lower significance threshold. The localisation of the cerebellar activity differed slightly in each subject (Table 1). Figure 3 shows the region of the right cerebellar cortex that showed a positive regression with delay using the combined data of all subjects. Right cerebellar cortex blood flow is plotted against delay, as recorded by the PC, in Fig. 4. No other brain regions showed a significant relationship with delay.

\section{DISCUSSION}

The present study sought to investigate how $\mathrm{rCBF}$ is modulated by parametric perturbations of the correspondence between self-generated movements and their sensory consequences. Activity in the right lateral cerebellar cortex (the border of VI and Crus I [21]) was positively correlated with delay between the movement of the right hand and the tactile stimulation on the left palm. Under all delays the right hand made the same movement and the left hand experienced the same stimulus. Only the temporal relationship between the action of the right hand and the sensory effect on the left hand was altered. It is proposed that as the delay increases the forward model's prediction of the sensory consequences of the movement becomes less accurate, and thus the sensory discrepancy between predicted and actual sensory feedback increases. Therefore, these results support the proposal that the cerebellar cortex is involved in signalling the sensory

Table I. Localisation of the cerebellar blood flow that showed a positive regression with delay in each subject.

\begin{tabular}{lll}
\hline Subject & Coordinates $(x, y, z)$ in cerebellum & Z value \\
\hline 1 & $40,-48,-34$ & 1.96 \\
2 & $40,-68,-40$ & 3.36 \\
3 & $50,-70,-50$ & 2.55 \\
4 & $10,-72,-42$ & 2.48 \\
5 & $36,-36,-50$ & 3.07 \\
6 & $34,-30,-20$ & 3.73 \\
\hline
\end{tabular}

discrepancy between predicted and actual sensory consequences of movements.

That activity in the right cerebellar cortex showed a positive correlation with delay is consistent with the theory that the cerebellum is a component of a system that provides precise predictions of the sensory consequences of motor commands $[12,13]$. The main input to the cerebellum, the climbing fibres from the inferior olive, has been proposed to act as a comparator between intended and achieved movement, signalling errors in motor performance and neurophysiological data [10]. Evidence for this comes from electrophysiological studies demonstrating that neurons in the inferior olive of cats respond to passively applied cutaneous stimuli but not to similar stimuli produced by a voluntary movement of the cat (except when stimuli were unexpectedly encountered during movement [9]).

Our data are also in accordance with previous functional imaging data demonstrating that various foci in the cerebellar cortex are activated during the early [11,14,15] and late phases of motor learning [15]. The region of the cerebellar cortex that was correlated with delay in the present study is strikingly similar to the cerebellar regions activated during the early stages of motor learning in two recent functional imaging studies (employing PET [22] and fMRI [15]). In terms of the forward model, activation of the cerebellum early in learning might correspond to the error signals between the predicted and actual outcomes of movements, which are used to refine the forward model's predictions and guide the acquisition of new internal models [16]. It is possible that the cerebellar activation found in the present study signals the sensory discrepancy, which is used to update the forward model's predictions according to the new contingency (the presence of a delay) between movement and its sensory consequences. However, it is unlikely that learning of the delays would occur in the present study for two reasons. First, the experiment was relatively short: many more trials would be required for learning of a time delay to take place [23]. Second, the delays were presented to the subjects in a random order. It
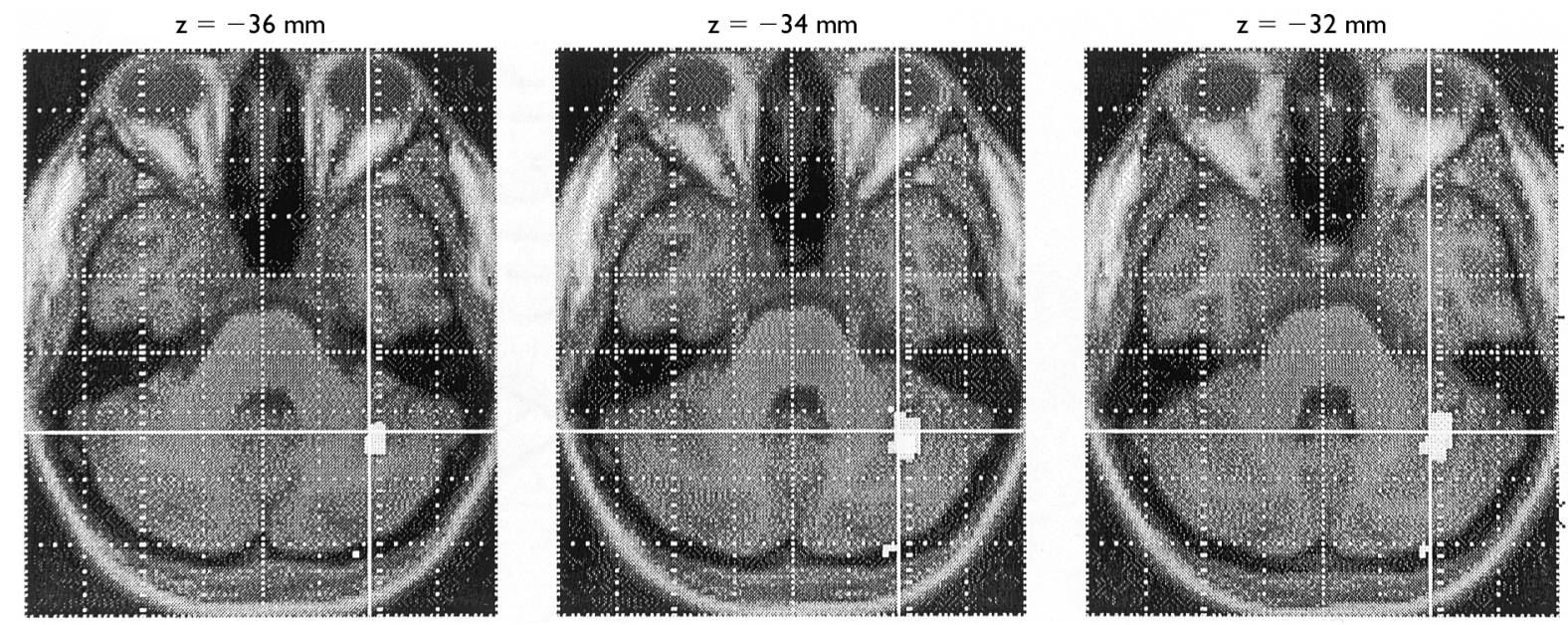

Fig. 3. Axial slices through the region of the middle right cerebellar cortex $(38,-56,-34$; VI/Crus $\mathrm{I})$ that showed a significant $(p<0.05)$ positive relationship with actual delay in all subjects combined when movement frequency and amplitude were used as confounds in the analysis. 


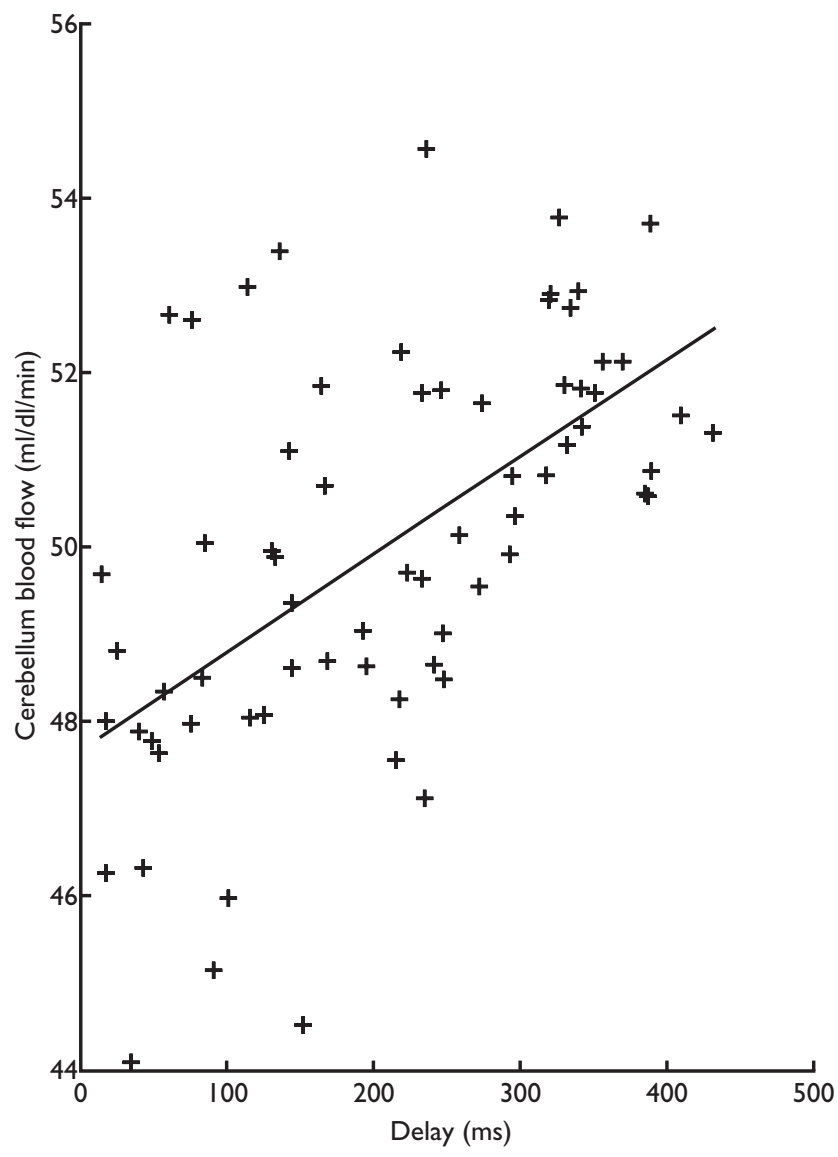

Fig. 4. Graph to show the significant $(p<0.05)$ positive relationship between blood flow in the middle right cerebellar cortex $(38,-56,-34$; $\mathrm{VI} /$ Crus I) and delay between the movement of the right hand and the tactile stimulus on the left palm in all subjects combined. The correlation coefficient between delay and cerebellar blood flow was $r=0.57$.

is likely that learning could only occur if the subject repeatedly experienced the same delay.

The region of the cerebellum activated in the present study is very close to a region activated in a PET study in which subjects lifted objects using a precision grip [24]. Forward model prediction is crucial for the production of anticipatory grip force modulation required to manipulate objects with speed and precision, which has been associated with the cerebellum [25]. The findings of Kinoshita et al. [24] support the suggestion that the right lateral cerebellar cortex is involved in processing the sensory predictions made by a forward model. Our data suggest the role of this area is to compare and signal the discrepancy between the sensory predictions and the actual sensory feedback of movement.

Cerebellar activity has previously been related to movement frequency [26] and amplitude [27]. However, both these movement parameters were recorded and removed as confounds in the analysis of the present study. Thus, the cerebellar activity observed in the present study did not relate to the frequency or amplitude of the subjects' movements, and must have related to the delay between the movements and their sensory consequences, as this was the only component of the paradigm that significantly changed between conditions.

One potential question concerns the lack of correlation between activity in the somatosensory cortex and delay. In our previous fMRI experiment, we found that the somatosensory cortex is more highly activated by tactile stimulation that was externally produced than by self-produced tactile stimulation [8]. However, the BOLD response in somatosensory cortex was attenuated by all self-generated movement: these areas were activated equally by movement that did result in tactile stimulation and by movement that did not. In other words the movement-related somatosensory gating did not seem to depend on the specific sensory consequences of the movement, but instead was associated with all self-generated movements. On the basis of these fMRI results it would be predicted that the somatosensory cortex would show the same level of activity as long as movement occurs. In the present study movement occurred in each condition so we would not predict that activity in the somatosensory cortex would correlate with delay.

In contrast, on the basis of the fMRI results we would predict that activity in the cerebellum would depend on the specific sensory consequences of movement. Our psychophysical experiment described above suggests that sensory gating is very sensitive to the consequences of the movement since very small delays modulated the perception of the tactile stimulus [5]. Our fMRI data suggested that the cerebellum might be involved in processing the specific sensory consequences of movement. In contrast to activity in somatosensory cortex, right lateral cerebellar cortex activity was not attenuated as a general consequence of all movement in our fMRI experiment. Instead, this area was differentially activated by self-produced movement that resulted in tactile stimulation and by self-produced movement alone. This pattern suggests that activity in the cerebellum depends on the specific sensory consequences of a movement, a hypothesis that is strongly supported by the current PET data.

\section{CONCLUSION}

This study was designed to test the hypothesis that the cerebellum is involved in predicting the sensory consequences of action, and in signalling the sensory discrepancy between the predicted and actual sensory feedback from actions. PET was used to examine neural responses to parametrically varied degrees of sensory discrepancy between the predicted and actual sensory consequences of movement. This was achieved by introducing varying degrees of delay between self-generated movement and its sensory consequences. As the tactile stimulus diverges temporally from the motor command producing it, the forward model's sensory prediction becomes less accurate, so the sensory discrepancy between the predicted and actual sensation increases. Activity in the right lateral cerebellar cortex showed a positive correlation with the delay between the movement of the right hand and the tactile stimulation on the left. This supports the proposal that the cerebellum is involved in signalling the sensory discrepancy resulting from the forward model's comparison between the predicted and actual sensory feedback from movements. 


\section{REFERENCES}

1. Frith CD. The Cognitive Neuropsychology of Schizophrenia. UK: Lawrence Erlbaum Associates; 1992.

2. Wolpert DM. Trends in Cognitive Science 1, 209-216 (1997).

3. Frith, CD, Blakemore S-J and Wolpert DM. Phil Trans Roy Soc Lond Biol Sci 355, 1771-1788 (2000).

4. Wolpert DM, Ghahramani Z and Jordan MI. Science 269, 1880-1882 (1995).

5. Blakemore S-J, Frith CD and Wolpert DW. J Cogn Neurosci 11, 551-559 (1999).

6. Weiskrantz L, Elliot J and Darlington C. Nature 230, 598-599 (1971).

7. Chapman CE. Can J Physiol Pharmacol 72, 558-570 (1994).

8. Blakemore S-J, Wolpert DM and Frith, CD. Nature Neurosci 1, 635-640 (1998).

9. Gellman R, Gibson AR and Houk JC. J Neurophysiol 54, 40-60 (1985).

10. Simpson JL, Wylie DR De Zeeuw CI. Behavioural and Brain Sciences 19, 384 (1995).

11. Imamizu H, Miyauchi S, Tamada T et al. Nature 403, 192-195 (2000).

12. Ito M. Int J Neurol 7, 162-176 (1970).

13. Miall RC, Weir DJ, Wolpert DM and Stein JF. J Mot Behav 25, 203-216 (1993).

14. Seitz RJ, Canavan AG, Yaguez L et al. Neuroreport 5, 2541-2544 (1994).
15. Tracy JI, Faro SS, Mohammed F et al. Brain Res Cogn Brain Res 10, 303-316 (2001).

16. Wolpert DM and Kawato M. Neural Networks 11, 1317-1329 (1998).

17. Friston KJ, Ashburner J, Frith CD et al. Human Brain Mapping 3, 165 (1995).

18. Talairach J and Tournoux P. Co-planar Stereotaxic Atlas of the Human Brain. New York: Thieme; 1988.

19. Friston KJ, Holmes AP, Worsley KJ et al. Human Brain Mapping 2, 189-210 (1995).

20. Worsley KJ, Marrett P, Neelin AC et al. Human Brain Mapping 4, 58-73 (1996).

21. Schmahmann JD, Doyon J, McDonald D et al. Neuroimage 10, 233-260 (1999).

22. Ghilardi M, Ghez C, Dhawan V et al. Brain Res 871, 127-145 (2000).

23. Witney AG, Goodbody SJ and Wolpert DM. J Neurophysiol 82, 2039-2048 (1999).

24. Kinoshita H, Oku N, Hashikawa K and Nishimura T. Brain Res 857, 119-130 (2000).

25. Tamada T, Miyauchi S, Imamizu H et al. Neuroimage 6, s492 (1999).

26. Jenkins IH, Passingham RE and Brooks DJ. J Neurol Sci 151, 195-205 (1997).

27. Waldvogel D, van Gelderen P, Ishii K and Hallett M. J Cerebr Blood Flow Metab 19, 1209-1212 (1999).

Acknowledgements: This work was supported by the Wellcome Trust and McDonnell Foundation. S-J.B. was supported by a Wellcome Trust 4 year PhD Programme in Neuroscience at University College London. 